Article

\title{
How Does Antimicrobial Stewardship Affect Inappropriate Antibiotic Therapy in Urological Patients?
}

\author{
Atsushi Uda ${ }^{1,2}$, Katsumi Shigemura ${ }^{1,3,4, *}$, Koichi Kitagawa ${ }^{3,5}$, Kayo Osawa ${ }^{3,6}{ }^{(}$, \\ Kenichiro Onuma ${ }^{1}$, Shigeaki Inoue ${ }^{7}$, Joji Kotani ${ }^{7}$, Yonmin Yan ${ }^{4}$, Yuzo Nakano ${ }^{4}$, \\ Tatsuya Nishioka ${ }^{2}$, Ikuko Yano ${ }^{2}$, Takayuki Miyara ${ }^{1}$ and Masato Fujisawa ${ }^{4}$ \\ 1 Department of Infection Control and Prevention, Kobe University Hospital, Kobe 650-0017, Japan; \\ a-uda@umin.ac.jp (A.U.); onumak@med.kobe-u.ac.jp (K.O.); miyarat@med.kobe-u.ac.jp (T.M.) \\ 2 Department of Pharmacy, Kobe University Hospital, Kobe 650-0017, Japan; tnishi@med.kobe-u.ac.jp (T.N.); \\ iyano@med.kobe-u.ac.jp (I.Y.) \\ 3 Division of Infectious Diseases, Department of Public Health, Kobe University Graduate School of Health \\ Sciences, Kobe 654-0142, Japan; ko1.kitgwa@gmail.com (K.K.); osawak@kobe-u.ac.jp (K.O.) \\ 4 Division of Urology, Kobe University Graduate School of Medicine, Kobe 650-0017, Japan; \\ yym1112@gmail.com (Y.Y.); yznakano@med.kobe-u.ac.jp (Y.N.); masato@med.kobe-u.ac.jp (M.F.) \\ 5 Division of Advanced Medical Science, Kobe University Graduate School of Science, \\ Technology and Innovation, Kobe 657-8501, Japan \\ 6 Department of Medical Technology, Kobe Tokiwa University, Kobe 653-0838, Japan \\ 7 Department of Disaster and Emergency Medicine, Kobe University Graduate School of Medicine, \\ Kobe 650-0017, Japan; inoues@med.kobe-u.ac.jp (S.I.); kotanijo0412@gmail.com (J.K.) \\ * Correspondence: katsumi@med.kobe-u.ac.jp; Tel.: +81-78-382-6155
}

Received: 19 January 2020; Accepted: 3 February 2020; Published: 6 February 2020

check for updates

\begin{abstract}
Antimicrobial stewardship teams (ASTs) have been well-accepted in recent years; however, their clinical outcomes have not been fully investigated in urological patients. The purpose of this study was to evaluate the outcomes of intervention via a retrospective review of urological patients, as discussed in the AST meetings, who were treated with broad-spectrum antibiotics between 2014 and 2018 at the Department of Urology, Kobe University Hospital in Japan. Interventions were discussed in AST meetings for patients identified by pharmacists as having received inappropriate antibiotic therapy. The annual changes in numbers of inappropriate medications and culture submissions over five years at the urology department were statistically analyzed. Among 1,033 patients audited by pharmacists, inappropriate antibiotic therapy was found in 118 cases $(11.4 \%)$. The numbers of inappropriate antibiotic use cases and of interventions for indefinite infections had significantly decreased during the study period ( $p=0.012$ and $p=0.033$, respectively). However, the number of blood and drainage culture submissions had significantly increased ( $p=0.009$ and $p=0.035$, respectively). Our findings suggest that urologists have probably become more familiar with infectious disease management through AST intervention, leading to a decrease in inappropriate antibiotic use and an increase in culture submissions.
\end{abstract}

Keywords: antimicrobial stewardship team; intervention; urological patient

\section{Introduction}

The main goal of antimicrobial stewardship (AS) is to decrease the incidence of antimicrobial resistance (AMR) [1]. Healthcare professionals and even national governments have called for action to prevent the increase of AMR by the appropriate use of broad-spectrum antibiotics, such as carbapenems, 
antibiotics with anti-Pseudomonas activity and anti-methicillin-resistant Staphylococcus aureus (MRSA) agents, based on reliable publications or guidelines. The concept of AS has spread globally, and the Japanese government has recently offered an additional medical fee for hospitals having the established AS team (AST) system [2]. Basically, the AST consists of physicians, pharmacists, clinical microbiologists, and nurses specialized in infectious disease and antimicrobial agents [3]. They are often certified infection control specialists such as infection control doctors (ICDs). Most ICDs are internal medical doctors (physicians) who are familiar with medications, but not with surgical interventions such as drainages [4]. Therefore, surgeons need to be included in the ASTs as well as in infection control teams (ICTs), to achieve the best outcomes for surgical patients [5]. Especially in the case of urinary tract infection (UTI), physicians often make late decisions to carry out drainage or use of invalid antibiotics that possibly generate resistance in bacteria. Furthermore, as urologists sometimes use invalid broad-spectrum antibiotics, collaboration among several professionals in ASTs is urgently needed. This study investigated patients, who were admitted and treated with broad-spectrum antibiotics in the urological ward, and compared the changes in the numbers of inappropriately prescribed medications and culture submissions over five years.

\section{Materials and Methods}

\subsection{Patients}

The present study included infectious disease patients admitted in the urological ward in Kobe University Hospital, who were discussed in the AST meetings held between 2014 and 2018. In our AST meetings, these patients were reviewed for the appropriateness of broad-spectrum antibiotic use, including antibiotic medication or whether cultures had to be obtained for antibiotic selection. This study was approved by the Kobe University Graduate School of Health Sciences Institutional Review Board (IRB) (No. 472-5).

\subsection{Antimicrobial Stewardship Team (AST) Meetings}

In March 2010, Kobe University Hospital started AST meetings which was called as the "Big Gun Project" in our hospital and focused on weekly prospective audit and feedback [6]. The main objectives of this project team were to promote the appropriate use of antimicrobial agents and to prevent the emergence of AMR. We defined antipseudomonal antibiotics and anti-MRSA agents as targeted, intravenous, broad-spectrum antibiotics in this meeting. Pharmacists in the AST audited all patients who used targeted antimicrobial agents in the previous week, and inappropriate (unnecessary) use or unsubmited culture cases were extracted for intervention. When the cases were discussed in the AST meeting, pharmacists recommended pharmaceutical interventions, such as another antimicrobial agent, optimization of the duration of administration or culture submissions, to the doctors. In these AST meetings, pharmacists, clinical microbiologists, nurses, and infectious disease physicians, including a urologist, collaborated to optimize the use of broad-spectrum antimicrobial agents for each patient. We found that this project was highly effective in reducing the use of antipseudomonal antibiotics [6].

\subsection{Infectious Diseases and Reasons for Intervention}

The medical records of patients, who were discussed in the AST meeting, were examined for urological infectious diseases and reasons for intervention. The types of disease that required drainage were as follows: pyelonephritis, peritonitis, refractory urachal abscess, renal abscess, appendicitis with abscess, perineum and penile abscess, pelvic abscess, Fournier's gangrene, pneumonia or liver abscess, renal cyst, and retroperitoneal abscess. Diseases other than the aforementioned ones had only one case during the entire study period. 


\subsection{Antibiotics Prescribed}

The AST investigated the following intravenous antibiotics in the meetings: (1) Antipseudomonal penicillins - piperacillin and piperacillin/tazobactam; (2) Monobactam-aztreonam; (3) Antipseudomonal cephalosporins - ceftazidime, cefepime, and cefozopran; (4) Carbapenems - meropenem, and doripenem; (5) Fluoroquinolones_ciprofloxacin, levofloxacin, and pazufloxacin; (6) Aminoglycosides—amikacin, tobramycin, and gentamicin; (7) Anti-MRSA agents-vancomycin, teicoplanin, linezolid, and daptomycin; (8) Other-colistin.

\subsection{Microbiological Culture Collections}

The data for blood, urine, or drainage culture collections of the patients admitted in the urological ward were obtained from microbiology laboratory records (blood culture sets/1000 patient-days, urine or drainage cultures/1000 patient-days).

\subsection{Statistical Analysis}

Appropriateness of cases, disease, selected antimicrobials, reasons for intervention, and microbiological cultures for each year were compared across a five-year period. Single regression analysis was performed to determine the association between the years and each data category by using EZR (Saitama Medical Center, Jichi Medical University, Saitama, Japan). In case of any change in the number of patients between the years, Spearman's correlation coefficient by rank was used. Statistical analysis was performed if more than 9 cases were included. The threshold for statistical significance was set at $p<0.05$.

\section{Results}

\subsection{Patients}

From January 2014 to December 2018, a total of 1033 urological inpatients, who were treated with broad-spectrum antimicrobials, were audited by pharmacists. Of these 1033 patients, 118 patients (106 male and 12 female), who were identified as having received inappropriate antibiotics, were discussed in the AST meetings. Figure 1 shows the percentage of inappropriate antibiotic cases identified by the pharmacists from the total count of urological patients each year. The percentage of cases identified as having used antibiotics inappropriately significantly decreased during the study period $(p=0.012)$.

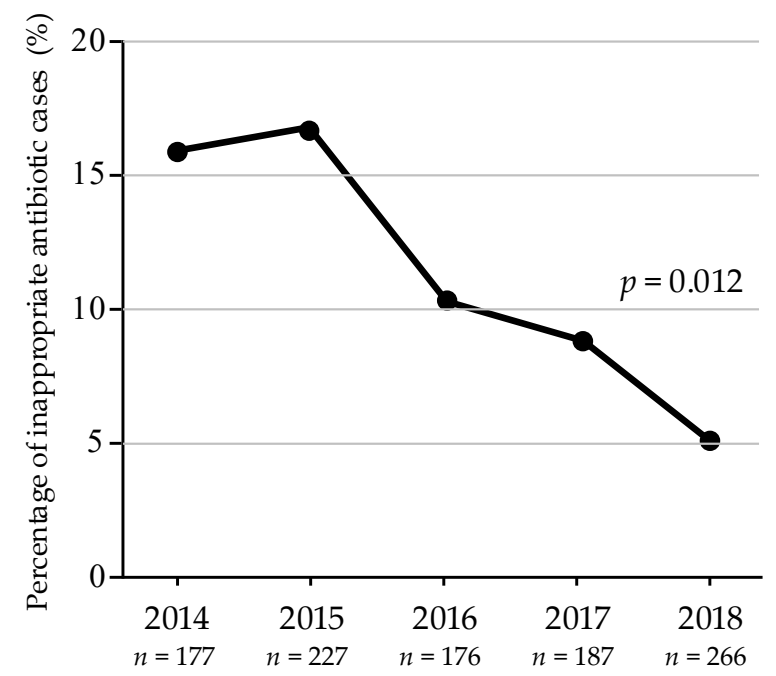

Figure 1. The percentage of inappropriate antibiotic cases among the patients audited by pharmacists between 2014 and 2018 . 
Table 1 shows the details of infectious diseases in 118 patients. The most common diseases were pyelonephritis $(n=55,46.6 \%)$, followed by indefinite infection $(n=18,15.3 \%)$, febrile neutropenia $(n=14,11.9 \%)$, and wound infection $(n=5,4.2 \%)$. Of the 118 patients discussed in the AST meetings, 28 patients $(23.7 \%)$ were assessed as requiring drainage by urologists. Statistical analyses showed that the number of indefinite infections significantly decreased over a five-year period $(p=0.033)$.

Table 1. Urological infectious diseases discussed in the antimicrobial stewardship teams (AST) meetings from 2014 to 2018.

\begin{tabular}{|c|c|c|c|c|c|c|c|}
\hline Infectious disease & $\begin{array}{c}2014-2018 \\
(n=118)\end{array}$ & $\begin{array}{c}2014 \\
(n=29)\end{array}$ & $\begin{array}{c}2015 \\
(n=39)\end{array}$ & $\begin{array}{c}2016 \\
(n=19)\end{array}$ & $\begin{array}{c}2017 \\
(n=17)\end{array}$ & $\begin{array}{c}2018 \\
(n=14)\end{array}$ & $p$ \\
\hline $\begin{array}{l}\text { Disease that require } \\
\text { drainage }\end{array}$ & 28 & 4 & 7 & 8 & 5 & 4 & 0.87 \\
\hline Pyelonephritis & 55 & 9 & 15 & 10 & 10 & 11 & 0.63 \\
\hline Indefinite infection & 18 & 6 & 6 & 3 & 1 & 2 & 0.033 \\
\hline Febrile neutropenia & 14 & 1 & 9 & 4 & 0 & 0 & 0.17 \\
\hline Wound infection & 5 & 2 & 1 & 1 & 1 & 0 & - \\
\hline s/o peritonitis & 4 & 4 & 0 & 0 & 0 & 0 & - \\
\hline Acute bacterial prostatitis & 3 & 1 & 0 & 2 & 0 & 0 & - \\
\hline CRBSI & 2 & 1 & 1 & 0 & 0 & 0 & - \\
\hline Bacteremia & 2 & 2 & 0 & 0 & 0 & 0 & - \\
\hline Acute prostatitis & 2 & 0 & 2 & 0 & 0 & 0 & - \\
\hline Others & 16 & 3 & 5 & 1 & 6 & 1 & 0.74 \\
\hline Total cases & $121^{a}$ & 29 & 39 & 21 & 18 & 14 & \\
\hline
\end{tabular}

s/o, suspect of; CRBSI, catheter-related blood stream infection. ${ }^{a}$ As some patients had $>1$ infectious disease, the total count exceeded 118.

Table 2 shows the antibiotics discussed in the meetings; other antibiotics not listed in Table 2 were not prescribed for urological patients. Antipseudomonal penicillin $(n=67,56.8 \%)$ was most frequently prescribed, followed by carbapenems $(n=25,21.2 \%)$, antipseudomonal cephalosporins $(n=16,13.6 \%)$, and anti-MRSA antibiotics $(n=10,8.5 \%)$. The number of cases who had been prescribed antipseudomonal penicillin inappropriately significantly decreased $(p=0.017)$.

Table 2. Antibiotics prescribed for patients listed in Table 1.

\begin{tabular}{|c|c|c|c|c|c|c|c|}
\hline Antibiotics & $\begin{array}{c}2014-2018 \\
(n=118)\end{array}$ & $\begin{array}{c}2014 \\
(n=29)\end{array}$ & $\begin{array}{c}2015 \\
(n=39)\end{array}$ & $\begin{array}{c}2016 \\
(n=19)\end{array}$ & $\begin{array}{c}2017 \\
(n=17)\end{array}$ & $\begin{array}{c}2018 \\
(n=14)\end{array}$ & $p$ \\
\hline $\begin{array}{l}\text { Antipseudomonal } \\
\text { penicillins }\end{array}$ & 67 & 18 & 16 & 13 & 12 & 8 & 0.017 \\
\hline Carbapenems & 25 & 4 & 9 & 3 & 4 & 5 & 0.94 \\
\hline $\begin{array}{l}\text { Antipseudomonal } \\
\text { cephalosporins }\end{array}$ & 16 & 7 & 7 & 1 & 0 & 1 & 0.11 \\
\hline Anti-MRSA agents & 10 & 1 & 6 & 2 & 0 & 1 & 0.49 \\
\hline Total cases & $122^{a}$ & 30 & 39 & 20 & 17 & 16 & \\
\hline
\end{tabular}

${ }^{\text {a }}$ As some patients had $>1$ prescribed antibiotic, the total count exceeded 118 .

\subsection{Reasons for Intervention}

Table 3 shows the reasons for AST intervention. The representative reasons for intervention included the need for de-escalation $(n=35,29.7 \%)$, dose optimization $(n=25,21.2 \%)$, inappropriate selection of antibiotics $(n=20,16.9 \%)$, and patients for whom no cultures had been submitted $(n=11,9.3 \%)$. 
Table 3. Reasons for AST intervention.

\begin{tabular}{|c|c|c|c|c|c|c|c|}
\hline Reason for intervention & $\begin{array}{c}2014-2018 \\
(n=118)\end{array}$ & $\begin{array}{c}2014 \\
(n=29)\end{array}$ & $\begin{array}{c}2015 \\
(n=39)\end{array}$ & $\begin{array}{c}2016 \\
(n=19)\end{array}$ & $\begin{array}{c}2017 \\
(n=17)\end{array}$ & $\begin{array}{c}2018 \\
(n=14)\end{array}$ & $p$ \\
\hline De-escalation & 35 & 5 & 12 & 7 & 7 & 4 & 0.50 \\
\hline Dose optimization & 25 & 10 & 8 & 4 & 0 & 3 & 0.083 \\
\hline $\begin{array}{c}\text { Inappropriate selection of } \\
\text { antibiotics }\end{array}$ & 20 & 5 & 6 & 4 & 3 & 2 & 0.083 \\
\hline $\begin{array}{l}\text { No cultures submitted for } \\
\text { pathogen identification }\end{array}$ & 11 & 6 & 3 & 0 & 1 & 1 & 0.17 \\
\hline $\begin{array}{l}\text { Duration of antimicrobial } \\
\text { therapies }\end{array}$ & 10 & 0 & 2 & 0 & 5 & 3 & 0.17 \\
\hline Escalation & 4 & 3 & 1 & 0 & 0 & 0 & - \\
\hline Unknown focus & 2 & 0 & 0 & 0 & 1 & 1 & - \\
\hline Others & 20 & 6 & 5 & 5 & 2 & 2 & $<0.001$ \\
\hline Total cases & $127^{\mathrm{a}}$ & 35 & 37 & 20 & 19 & 16 & \\
\hline
\end{tabular}

a As some patients had $>1$ reason, total count exceeded 118

\subsection{Microbiological Culture Collections}

Table 4 shows blood, urine, or drainage culture collections during the study period. Blood and drainage culture collections significantly increased during the study period $(p=0.009$ and $p=0.035$, respectively).

Table 4. Microbiological culture collections (blood culture sets/1000 patient-days, urine or drainage cultures/1000 patient-days).

\begin{tabular}{ccccccc}
\hline Sample Type & $\mathbf{2 0 1 4}$ & $\mathbf{2 0 1 5}$ & $\mathbf{2 0 1 6}$ & $\mathbf{2 0 1 7}$ & $\mathbf{2 0 1 8}$ & $\boldsymbol{p}$ \\
\hline Blood culture & 15.8 & 19.9 & 22.3 & 22.3 & 26.6 & 0.009 \\
Urine culture & 14.4 & 25.9 & 35.4 & 36.1 & 27.4 & 0.24 \\
Drainage culture & 0.11 & 0.46 & 0.39 & 0.51 & 0.89 & 0.035 \\
\hline
\end{tabular}

\section{Discussion}

The AS concept is spreading nationwide in Japan, partly due to the AMR National Action Plan [7]. The concept aims at controlling antimicrobial use, decreasing the number of AMR bacterial strains and, importantly, reducing the unnecessary use of broad-spectrum antibiotics [8]. In collaboration with ICDs, the AST plays a major role in managing antimicrobial use to control infectious diseases due to AMR bacterial strains.

AST and ICD interventions have been studied before. Honda et al. reported that the initiation of adequate empiric antimicrobial therapy had important implications for AS even in the elderly population in Japan [9]. Other studies have also discussed AST and infectious disease control from the point of view of physicians, pharmacists, or clinical microbiologists [6,10-12].

Urologists have an important role in infectious disease management of patients with UTI, urosepsis, or drainage for obstructive disease such as a stone-related obstructive UTI. To assess the indications for drainage or stenting in the urinary tract, ASTs need to collaborate with surgeons, including urologists [13]. They can decide to carry out drainage using ureter stents, urethral catheters, or renal fistulas, as necessary, and ensure that they do not miss an opportunity to collect culture and choose the appropriate antibiotics. Proper drainage improves renal function, and improved renal function makes antibiotic treatment more effective [14]. The timing of surgical intervention should not be delayed by performing ineffective, empiric antibiotic treatments for infection, which is one of the risk factors for lower survival in sepsis [15].

Our data demonstrate that intervention by the AST significantly decreased both the percentage of inappropriate antibiotic use cases and the number of cases for intervention, specifically, the number of indefinite infections over the five-year study period ( $p=0.012$ and $p=0.033$, respectively). Usually, 
this kind of study tends to be conducted in an internal medicine department where infectious disease management tends to be limited to the selection of an antimicrobial regimen and microbial laboratory examination. However, infectious diseases should be often treated by drainage, including surgical drainage performed by surgeons. Recently, studies conducted by urological departments have been published. Spooremberg et al. demonstrated that an earlier switch from intravenous to oral treatment led to more favorable patient outcomes and lower healthcare costs [16]. Doernberg, et al. conducted quasi-experimental historical controls with a six-month retrospective period versus a six-month intervention period for asymptomatic bacteriuria and UTI in patients in a long-term care facility setting, with 104 antibiotic prescriptions for UTI. They demonstrated: (1) weekly prospective audit and feedback from the AST over a six-month period resulted in decreased antibiotic use, (2) many lost opportunities for intervention were identified, and (3) no significant effect was noted on resistant organisms or detection of Clostridioides difficile [17]. Dik et al. studied an AST intervention cohort vs. a historic control cohort by time series analysis for antibiotic treatments in urological wards (114 intervention and 357 control cases) and showed significant reduction of antimicrobial consumption for all patients, decreased length of hospital stay, and an unchanged outcome for patients with severe underlying diseases, such as cancer [18]. The AST recommended antimicrobial treatment adjustment/de-escalation to appropriate therapy in the majority of bacteremic UTI patients, and the results showed a significantly lower mortality rate in de-escalation groups compared to those with no antimicrobial change [19]. AST intervention was an independent variable related to clinical cure, but no economic impact was seen in a retrospective study of UTI patients with extended spectrum beta-lactamase (ESBL)-producing bacteria [20]. Another retrospective cohort study of complicated UTI cases demonstrated significant reduction in the duration of antibiotic treatment and reduced length of hospitalization [21]. A representative review by a urological AST concluded that well-designed ASTs help reduce treatment duration, time-to-switching-to oral-antibiotics, and total antibiotic prescription, with an optimal assessment timing of approximately $24-48 \mathrm{~h}$. AST programs are also useful for the education and feedback for physicians [22].

We recommend that ASTs need to include urologists or surgeons to help avoid biases towards considering antimicrobial selection only for intervention and focusing on only broad-spectrum agents, such as carbapenems, rather than on surgical intervention. These biases negatively affect antimicrobial use and possibly patient outcomes, especially in surgical departments.

There are several limitations of the present study. First, this is a retrospective study with a small number of cases. Second, we had no available data on drainage procedures such as urinary tract stenting or percutaneous drainage. Third, no control groups were included. These limitations will be addressed in our future studies.

\section{Conclusions}

Our study was conducted with a well-established AST over five years [6]. Statistical analyses showed that the percentage of cases of inappropriate broad-spectrum antibiotic use and interventions for indefinite infections had significantly decreased, whereas the number of blood or drainage culture submissions had significantly increased over the five-year study period. These results suggest that urologists have probably become more familiar with antibiotic treatments based on evidence such as microbiological culture results. AST intervention might help to prevent inappropriate antibiotic use in surgical departments. Further studies are necessary for follow-up.

Author Contributions: Conceptualization, A.U. and K.S.; methodology, A.U.; software, K.K.; validation, K.K., K.O. (Kayo Osawa) and Y.Y.; formal analysis, A.U., K.O. (Kayo Osawa) and I.Y.; investigation, A.U., K.K. and K.O. (Kayo Osawa); resources, Y.N., S.I. and T.N.; data curation, A.U. and K.K.; writing-original draft preparation, K.S. and A.U.; writing-review and editing, A.U., K.S. and I.Y.; visualization, K.O. (Kenichiro Onuma); supervision, J.K. I.Y., and M.F.; project administration, T.M.; funding acquisition, T.M. All authors have read and agreed to the published version of the manuscript.

Funding: This research received no external funding. 
Acknowledgments: We thank Drs Issei Tokimatsu, Soichi Arakawa and Goh Ohji for patient care and AST meeting management.

Conflicts of Interest: The authors declare no conflict of interest.

\section{References}

1. Government of Japan. National Action Plan on Antimicrobial Resistance (AMR) 2016-2020. Ministry of Health, Labour \& Welfare Website. 2016. Available online: https://www.mhlw.go.jp/file/06-Seisakujouhou10900000-Kenkoukyoku/0000138942.pdf (accessed on 10 August 2019).

2. Saito, H.; Noda, H.; Takakura, S.; Jindai, K.; McLellan, R.T.; Asanuma, K. First practical major step toward appropriate antimicrobial use by the Government of Japan. Jpn. J. Infect. Dis. 2019, 72, 56-57. [CrossRef] [PubMed]

3. Morikane, K. Infection control in healthcare settings in Japan. J. Epidemiol. 2012, 22, 86-90. [CrossRef] [PubMed]

4. Tarchini, G.; Liau, K.H.; Solomkin, J.S. Antimicrobial stewardship in surgery: Challenges and opportunities. Clin. Infect. Dis. 2017, 64 (Suppl. 2), S112-S114. [CrossRef]

5. Deguchi, T.; Matsumoto, T. Antimicrobial stewardship in urology. Int. J. Urol. 2014, 21, 628-629. [CrossRef] [PubMed]

6. Kimura, T.; Uda, A.; Sakaue, T.; Yamashita, K.; Nishioka, T.; Nishimura, S.; Ebisawa, K.; Nagata, M.; Ohji, G.; Nakamura, T.; et al. Long-term efficacy of comprehensive multidisciplinary antibiotic stewardship programs centered on weekly prospective audit and feedback. Infection 2018, 46, 215-224. [CrossRef]

7. Ohmagari, N. National Action Plan on Antimicrobial Resistance (AMR), Japan, 2016-2020. Nihon Naika Gakkai Zasshi 2017, 106, 2259-2264. [CrossRef]

8. Garau, J.; Nicolau, D.P.; Wullt, B.; Bassetti, M. Antibiotic stewardship challenges in the management of community-acquired infections for prevention of escalating antibiotic resistance. J. Glob. Antimicrob. Resist. 2014, 2, 245-253. [CrossRef]

9. Honda, H.; Higuchi, N.; Shintani, K.; Higuchi, M.; Warren, D.K. Inadequate empiric antimicrobial therapy and mortality in geriatric patients with bloodstream infection: A target for antimicrobial stewardship. J. Infect. Chemother. 2018, 24, 807-811. [CrossRef]

10. Zhang, C.; Li, S.; Ji, J.; Shen, P.; Ying, C.; Li, L.; Xiao, Y. The professional status of infectious disease physicians in China: A nationwide cross-sectional survey. Clin. Microbiol. Infect. 2018, 24, 82.e5-82.e10. [CrossRef]

11. Molloy, L.; McGrath, E.; Thomas, R.; Kaye, K.S.; Rybak, M.J. Acceptance of pharmacist-driven antimicrobial stewardship recommendations with differing levels of physician involvement in a children's hospital. Clin. Pediatr. 2017, 56, 744-751. [CrossRef]

12. Morency-Potvin, P.; Schwartz, D.N.; Weinstein, R.A. Antimicrobial stewardship: How the microbiology laboratory can right the ship. Clin. Microbiol. Rev. 2017, 30, 381-407. [CrossRef]

13. Shigemura, K.; Arakawa, S.; Fujisawa, M. Treatment strategies for urinary tract infections under consideration of antibiotic resistant strains: In terms of urologists. Jpn. J. Chemother. 2015, 63, 462-468.

14. Karl, I.E. The pathophysiology and treatment of sepsis. N. Engl. J. Med. 2003, 348, 138-150.

15. Micek, S.T.; Hampton, N.; Kollef, M. Risk factors and outcomes for ineffective empiric treatment of sepsis caused by gram-negative pathogens: Stratification by onset of infection. Antimicrob. Agents Chemother. 2018, 62, e01577-17. [CrossRef] [PubMed]

16. Spoorenberg, V.; Hulscher, M.E.; Akkermans, R.P.; Prins, J.M.; Geerlings, S.E. Appropriate antibiotic use for patients with urinary tract infections reduces length of hospital stay. Clin. Infect. Dis. 2013, 58, 164-169. [CrossRef] [PubMed]

17. Doernberg, S.B.; Dudas, V.; Trivedi, K.K. Implementation of an antimicrobial stewardship program targeting residents with urinary tract infections in three community long-term care facilities: A quasiexperimental study using time-series analysis. Antimicrob. Resist. Infect. Control 2015, 4, 54. [CrossRef] [PubMed]

18. Dik, J.W.H.; Hendrix, R.; Lo-Ten-Foe, J.R.; Wilting, K.R.; Panday, P.N.; van Gemert-Pijnen, L.E.; Leliveld, A.M.; van der Palen, J.; Friedrich, A.W.; Sinha, B. Automatic day-2 intervention by a multidisciplinary antimicrobial stewardship-team leads to multiple positive effects. Front. Microbiol. 2015, 6, 546. [CrossRef] 
19. Yanai, M.; Ogasawara, M.; Hayashi, Y.; Suzuki, K.; Takahashi, H.; Satomura, A. Impact of interventions by antimicrobial stewardship program team on appropriate antimicrobial therapy in patients with bacteremic urinary tract infection. J. Infect. Chemother. 2018, 24, 206-211. [CrossRef]

20. Esteve-Palau, E.; Grau, S.; Herrera, S.; Sorli, L.; Montero, M.; Segura, C.; Durán, X.; Horcajada, J.P. Impact of an antimicrobial stewardship program on urinary tract infections caused by extended-spectrum beta-lactamase-producing Escherichia coli. Rev. Esp. Quimioter. 2018, 31, 110.

21. Carbo, J.F.; Ruh, C.A.; Kurtzhalts, K.E.; Ott, M.C.; Sellick, J.A.; Mergenhagen, K.A. Male veterans with complicated urinary tract infections: Influence of a patient-centered antimicrobial stewardship program. Am. J. Infect. Control 2016, 44, 1549-1553. [CrossRef]

22. MacDougall, C.; Polk, R.E. Antimicrobial stewardship programs in health care systems. Clin. Microbiol. Rev. 2005, 18, 638-656. [CrossRef] [PubMed]

(C) 2020 by the authors. Licensee MDPI, Basel, Switzerland. This article is an open access article distributed under the terms and conditions of the Creative Commons Attribution (CC BY) license (http://creativecommons.org/licenses/by/4.0/). 